\title{
Long-term outcome of deep brain stimulation in generalised dystonia: a series of 60 cases
}

\author{
J J FitzGerald ${ }^{1,2} \mathrm{~F}$ Rosendal, ${ }^{1,3} \mathrm{~N}$ de Pennington, ${ }^{1} \mathrm{C}$ Joint, ${ }^{1} \mathrm{~B}$ Forrow, ${ }^{1} \mathrm{C}$ Fletcher, ${ }^{1}$ \\ A L Green, ${ }^{1,2}$ T Z Aziz ${ }^{1,2}$
}

'Department of Neurosurgery, John Radcliffe Hospital, Oxford, UK

${ }^{2}$ Nuffield Department of Surgical Sciences, University of Oxford, Oxford, UK ${ }^{3}$ Department of Neurosurgery, Aarhus University Hospital, Aarhus, Denmark

\section{Correspondence to} Dr James J FitzGerald, Department of Neurosurgery, Level 3, West Wing, John Radcliffe Hospital, Headington, Oxford OX3 9DU, UK; james. fitzgerald@nds.ox.ac.uk

Received 1 October 2013 Revised 17 January 2014 Accepted 8 March 2014 Published Online First 1 April 2014
CrossMark

\author{
To cite: FitzGerald JJ, \\ Rosendal $\mathrm{F}$, de \\ Pennington $\mathrm{N}$, et al. J \\ Neurol Neurosurg Psychiatry \\ 2014;85:1371-1376.
}

\begin{abstract}
Background There is solid evidence of the long term efficacy of deep brain stimulation of the globus pallidus pars interna in the treatment of generalised dystonia. However there are conflicting reports concerning whether certain subgroups gain more benefit from treatment than others. We analysed the results of a series of 60 cases to evaluate the effects of previously proposed prognostic factors including dystonia aetiology, dystonia phenotype, age at onset of dystonia, and duration of dystonia prior to treatment.

Methods 60 patients with medically intractable primary or secondary generalised dystonia were treated with deep brain stimulation of the globus pallidus pars interna during the period 1999-2010 at the Department of Neurosurgery in Oxford, UK. Patients were assessed using the Burke-Fahn-Marsden (BFM) Dystonia Rating Scale prior to surgery, 6 months after implantation and thereafter at 1 year, 2 years and 5 years follow-up. Results The group showed mean improvements in the BFM severity and disability scores of $43 \%$ and $27 \%$, respectively, by 6 months, and this was sustained. The results in 11 patients with DYT gene mutations were significantly better than in non-genetic primary cases. The results in 12 patients with secondary dystonia were not as good as those seen in non-genetic primary cases but there remained a significant beneficial effect. Age of onset of dystonia, duration of disease prior to surgery, and myoclonic versus torsional disease phenotype had no significant effect on outcome.
\end{abstract}

Conclusions The aetiology of dystonia was the sole factor predicting a better or poorer outcome from globus pallidus pars interna stimulation in this series of patients with generalised dystonia. However even the secondary cases that responded the least well had a substantial reduction in BFM scores compared with preoperative clinical assessments, and these patients should still be considered for deep brain stimulation.

\section{INTRODUCTION}

Dystonia is a movement disorder characterised by sustained involuntary muscle contractions leading to twisting movements and abnormal posturing. It may affect part of the body (eg, spasmodic torticollis) or, as in all the cases described here, it may be generalised. It is a painful and disfiguring disease that leaves the patient physically and socially disabled, and most types are refractory to all available medical treatments.

Generalised dystonia is not a single entity but rather a heterogeneous group of conditions with similar phenotypes but several different aetiologies.
In a minority of patients there is an identifiable cause such as traumatic brain injury, intrauterine/ perinatal insult or drug exposure, and in these cases the disease is classified as secondary dystonia. However the great majority of cases occurs in the absence of any such history and is therefore classified as primary. In some cases of primary dystonia an underlying genetic mutation can be found, the most common being mutation of the DYT1 gene. There are now over 20 identified DYT genes, ${ }^{1}$ but the majority of cases of primary generalised dystonia is not related to any of them and remains idiopathic.

Although the first case of deep brain stimulation (DBS) for dystonia was published in $1977,{ }^{2}$ only within the last 15 years has DBS of the globus pallidus pars interna (GPi) become a widely available treatment for medically refractory dystonia. It offers substantial improvement of motor function with a low risk of adverse effects, and the trials by Vidailhet et $a l^{3}$ and Kupsch et $a l^{4}$ have provided solid evidence of its efficacy in primary generalised and segmental dystonia. Further follow-up of these patients ${ }^{5}$ and the results of other case series ${ }^{7-13}$ demonstrate that the benefits are maintained long-term.

There have been several publications comparing the efficacy of GPi DBS among various subgroups of patients with dystonia. Some have reported that outcomes in generalised dystonia are significantly better in patients with DYT1 mutations than those without, ${ }^{11}{ }^{12}{ }^{14-18}$ while several others have not found this. ${ }^{4919}$ There is a general consensus that those with secondary dystonia do not do as well as primary cases. ${ }^{9} 162021$ It has also been reported that patients with shorter duration disease prior to treatment do better than those with longer. ${ }^{19} 2223$

We present here the outcomes of a consecutive series of 60 patients implanted with GPi DBS in the period 1999-2010 at the Department of Neurosurgery in Oxford. We look specifically at whether our data support the previously proposed patterns of response among different groups of patients, and whether there are any identifiable groups who may not benefit from what is generally agreed to be a highly effective treatment for the majority of patients with dystonia.

\section{METHODS}

\section{Clinical evaluation}

All patients being considered for surgery were evaluated by a consultant functional neurosurgeon and consultant neurologist, both specialists in movement disorders. The patients met the criteria for dystonia 
outlined by Fahn. ${ }^{24}$ Patients accepted for surgery had: (1) a normal neurological examination apart from the dystonic features; (2) significant functional disability; (3) failure of medical management including a trial of L-dopa to exclude Segawa's dystonia; (4) absence of untreated psychiatric disturbances or cognitive dysfunction; (5) absence of severe contractures and fixed skeletal deformities; and (6) absence of any medical contraindication to DBS surgery. Written, informed consent was obtained from each patient.

Objective clinical assessment was performed using the Burke-Fahn-Marsden Dystonia Rating Scale (BFMDRS). ${ }^{25}$ The BFMDRS severity score (referred to here as BFM-S) was based on standardised video recordings of motor examination, and the disability score (BFM-D) was based on interview with the patient. All patients had the BFMDRS score assessed prior to surgery and on each postoperative follow-up appointment. Assessments were performed by functional neurosurgery specialist nurses or clinical fellows trained in the use of the assessment tool.

Preoperatively, patients underwent MRI to exclude a structural lesion and for surgical planning. All patients were investigated for metabolic or other causes of secondary dystonia. Patients who had early onset dystonia, a family history of dystonia or clinical features suggestive of genetically induced dystonia were tested for DYT gene mutations. Finally the patients underwent neuropsychological assessment to exclude psychogenic dystonia and ensure the patient was cognitively suited to undergo DBS implantation.

\section{Surgery}

All patients had DBS implantation performed under general anaesthesia. A Cosman-Roberts-Wells (CRW) stereotactic frame was applied to the patient's head and a CT scan with $1 \mathrm{~mm}$ slice thickness performed. The CT scan was registered to a preoperatively obtained MRI scan (inversion recovery sequence) using ImageFusion software (Radionics, Massachusetts, USA) and target coordinates calculated using Stereoplan software (Radionics, Massachusetts, USA). The target was the posteroventral segment of the GPi. Model 3387 depth leads (Medtronic, Minneapolis, Minnesota) were used, aiming for the lead tip to be just superior to the optic tract, leaving the middle two contacts within the target area. Leads were inserted through $2.7 \mathrm{~mm}$ twist-drill holes, via tracts made using a rigid thermocouple (TC) electrode (Radionics, Massachusetts, USA) electrode, and secured to the skull with miniplates. Targeting was purely anatomical; microelectrode recording was not used. A repeat CT scan was performed and fused to the preoperative MRI to confirm the placement of the leads. An example of the postoperative imaging is shown in figure 1. Subsequently the implantable pulse generator (IPG) and extension wires were implanted and the IPG was programmed and activated. Patients were discharged and seen 4 weeks postoperatively for adjustment of IPG settings. At longterm follow-up mean stimulator voltages were $3.5 \mathrm{~V}$ (median $4.0 \mathrm{~V}$, IQR $2.7-4.2 \mathrm{~V}$ ) with frequencies in the range $130-180 \mathrm{~Hz}$ and pulse widths of 150-240 $\mu$ s.

\section{Data collection}

Standardised variables were prospectively recorded for each patient in a database. These included the patient's age at onset of symptoms, the duration of their dystonia prior to DBS, the aetiology type (primary or secondary), the presence or not of DYT mutations in primary dystonia, the cause in cases of secondary dystonia and the dystonia phenotype (torsional or myoclonic). BFMDRS scores were recorded at the preoperative evaluation and at follow-up appointments.
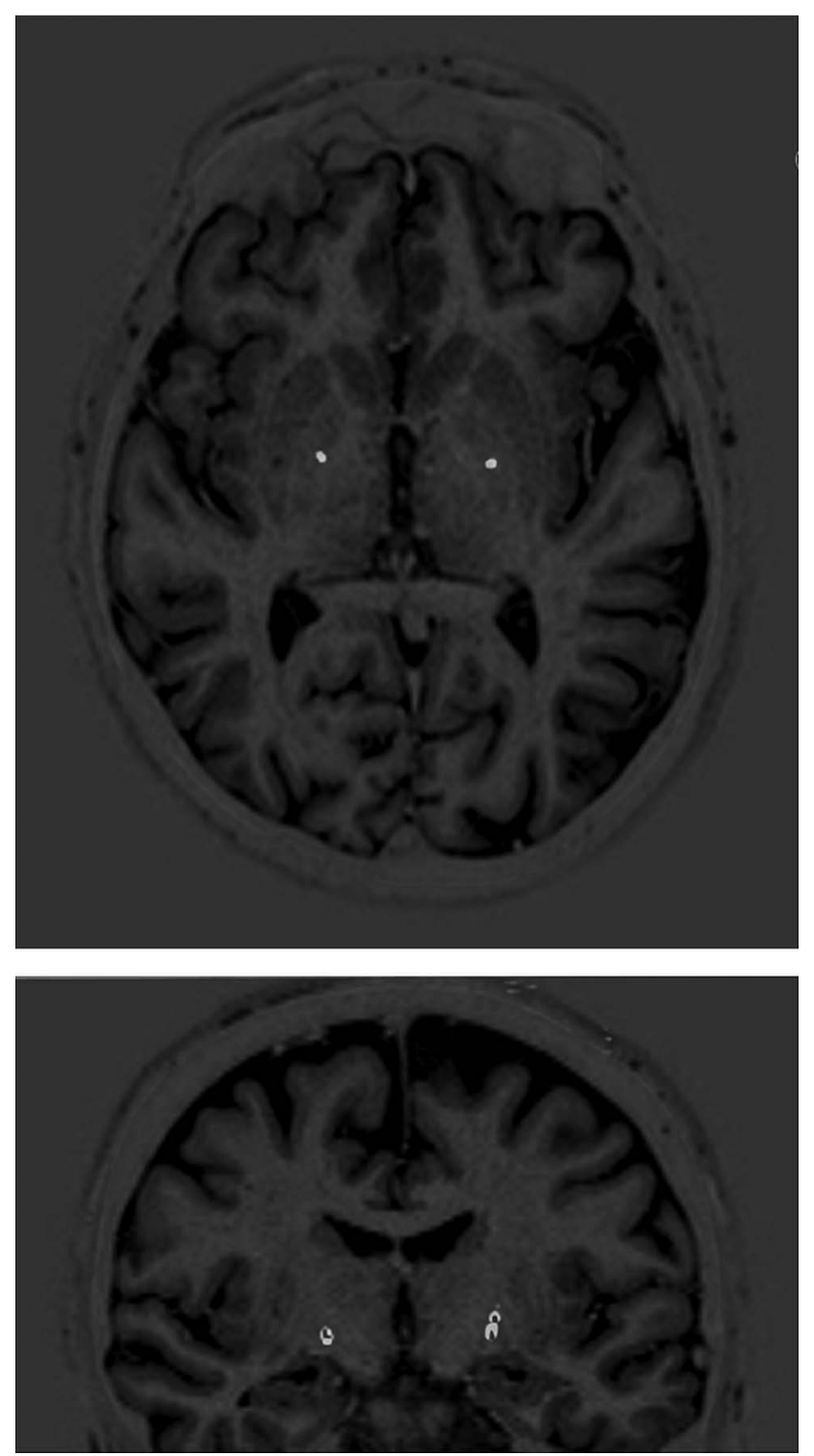

Figure 1 Representative example of a preoperative MRI (T1W inversion recovery) fused with a postoperative CT scan windowed to show only the leads (white). (In the coronal section only the lower end of the leads is seen as they run out of the plane of the slice).

\section{Statistical analysis and presentation of results}

Data were analysed using SPSS (IBM). A linear mixed model was used, treating preoperative and follow-up BFM-S and BFM-D scores as repeated measures. Age at onset, aetiology, phenotype and disease duration prior to treatment were included in the model as fixed variables. Age at onset was classified as paediatric (under 16 years old) or adult. Aetiology was classified as non-genetic primary, genetic or secondary. Phenotype was either torsional or myoclonic. Duration prior to treatment was categorised as less than 10 years, 10-20 years or over 20 years. Missing values of BFM-S and BFM-D were treated by multiple imputations. Interactions between the above putative predictor variables were tested for in the model and no significant interactions were found.

Some reports in the literature have expressed changes in BFM score after intervention as an absolute number of points from baseline, but the majority has expressed them as a percentage of the baseline. In our data set, absolute score improvements were 
strongly correlated with initial score (in line with the commonsense expectation that those who are the worst to start with have the greatest room for improvement). If the dependent variable of the model were absolute BFMDRS point reduction, initial score would have to be included in the model as a covariate. If however percentage improvement is taken as the dependent variable, there is no such dependence on initial score in our data. This makes the results easier for the reader to interpret and we have therefore adopted this approach.

The output of the mixed model is in the form of (A) an estimate of the treatment effect for a reference group (which we have chosen to be Patients with non-genetic primary dystonia, with torsional phenotype, presenting in childhood and treated less than 10 years from disease onset) and (B) how changes in putative predictor variables (eg, a genetic aetiology, myoclonic phenotype, longer disease duration prior to treatment, etc) affect this.

Graphs were plotted using Origin (OriginLabs).

\section{RESULTS}

Sixty-five patients with generalised dystonia were implanted with bilateral GPi DBS in the period 1999-2010 at the Department of Neurosurgery in Oxford, UK. Five patients were excluded from the study. Of these, two were excluded because of confounding surgical factors (one had a prior pallidotomy and the other had additional thalamic electrodes), two were excluded because no follow-up BFM scores were available and one patient was excluded because no preoperative BFM scores were available.

Table 1 summarises the clinical characteristics of the 60 patients analysed. Twenty-seven male and 33 female patients were included in this study (not a significant sex imbalance; $\mathrm{p}=0.44$ ). Forty-eight patients had primary generalised dystonia, of whom eight had DYT1 mutations, two DYT6 and one DYT11. Twelve were secondary: eight with cerebral palsy, three with progressive metabolic diseases (one each with an iron

Table 1 Summary of patient characteristics

\begin{tabular}{lc}
\hline Age at surgery & 33.5 \\
Mean & 32 \\
Median & \\
Sex & 27 \\
Male & 33 \\
Female & \\
Aetiology & \\
Primary & 37 \\
$\quad$ Non-genetic & 8 \\
DYT1 & 2 \\
DYT6 & 1 \\
DYT11 & 12 \\
Secondary & \\
Phenotype & 51 \\
Torsional & 9 \\
Myoclonic & \\
Age group at onset & 43 \\
Paediatric & 17 \\
Adult & \\
Duration pretreatment & \\
Up to 10 years & 14 \\
10-20 years & 23 \\
Over 20 years & 23 \\
\hline
\end{tabular}

deposition syndrome, pantothenate kinase-associated neurodegeneration and glutaric aciduria) and one with tardive dystonia. Nine patients presented with a myoclonic phenotype and 51 with a torsional phenotype. Mean age at onset of symptoms was 14.9 years (median 11 years, range $0-46$ years); in total 43 of the patients had paediatric disease onset, defined as symptoms occurring at 16 years of age or younger, while 17 presented in adulthood. Mean age at surgery was 33.5 years (median 32 years, range 7-69 years). The mean time period from disease onset to surgery was 19.1 years (median 15 years, range 262 years).

\section{Adverse events}

The adverse events in this series are summarised in table 2. The most common problem was infection (eight patients), all of which occurred at the time of IPG replacement rather than at primary surgery. One infection was cleared by a 6 month course of antibiotics; the other seven required removal of hardware. In two of these cases where dystonia was very severe and infection was limited to the IPG pocket, the patient was treated by temporary externalisation of the IPG and subsequent replacement of extensions and IPG once the infection had been eradicated. ${ }^{26}$ Six patients with suboptimal lead positioning underwent lead revision because of poor response (three patients) or stimulation related side effects (three patients). Lead fracture occurred in three patients, one of whom was having frequent falls. There were single cases of lead erosion in a patient with a thin scalp, and of a wound granuloma. One patient with an exhausted battery presented in status dystonicus requiring a period of intensive care.

\section{Overall outcome}

Figure 2 shows the mean BFM-S and BFM-D scores for the entire group at baseline and during follow-up. The mean improvement in BFM-S was $43.3 \%$ at 6 months, $43.5 \%$ at 1 year, $49.9 \%$ at 2 years and $49.8 \%$ at 5 years. The mean improvement in BFM-D was $27.0 \%$ at 6 months, $33.0 \%$ at 1 year, $35.2 \%$ at 2 years and $33.0 \%$ at 5 years. The reductions in BFM-S and BFM-D were significant at all time points with respect to baseline, with $\mathrm{p}<0.001$ (Mann-Whitney U test).

Figure 3 shows the responses to treatment of individual cases of non-genetic primary dystonia (left), dystonia associated with a DYT mutation (centre) and secondary dystonia (right). The three patients with DYT6 or DYT11 mutations performed similarly to the eight patients with DYT1 (mean improvements in BFM-S at 1 year of $52 \%$ for the three DYT6/ DYT11 cases vs $49 \%$ for the eight DYT1 cases, Mann-Whitney U test $\mathrm{p}=0.50$ ). The genetic cases were therefore analysed as one group.

The results of the mixed-model analysis are summarised in table 3. For patients with non-genetic primary dystonia, with childhood onset of disease and torsional phenotype, who are treated within 10 years of disease onset, there is a mean

\section{Table 2 Adverse events}

Infection

Lead revision

Lead fracture

Lead erosion through scalp

Wound granuloma

Status dystonicus 

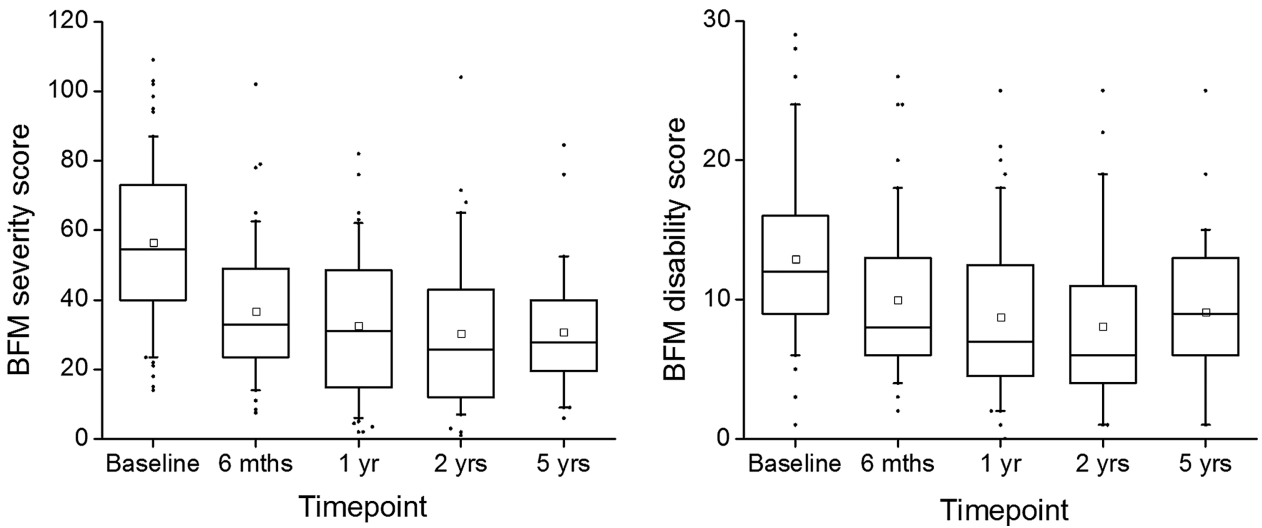

Figure 2 Overall outcome for entire group. Boxes: 25th centile, median and 75th centile. Whiskers: 10th and 90th centiles; points outside this are plotted individually. Small squares represent means.

improvement at 6 months of $40.3 \%$ in BFM-S $(\mathrm{p}<0.001)$ and $28.0 \%$ in BFM-D $(\mathrm{p}=0.003)$.

Compared with the results at 6 months, there is no significant change in either BFM-S or BFM-D at any later follow-up time point. This indicates that the results of treatment are (A) manifested within 6 months and (B) durable to at least 5 years.

\section{Effect of aetiology}

Patients with a DYT gene mutation showed a significantly better response to DBS than patients with primary dystonia without a genetic aetiology. Genetic mutation conferred an extra $14.8 \%$ reduction in BFM-S $(p=0.025)$ and an extra $20.7 \%$ reduction in BFM-D ( $\mathrm{p}=0.031)$.

Compared with patients with non-genetic primary dystonia, those with secondary dystonia tended to respond less well but this trend was not statistically significant for either BFM-S or BFM-D. However, as can be seen in figure 3, there was one patient who demonstrated a $96 \%$ improvement in BFM-S with DBS. This was the single patient with tardive dystonia, and such patients are known to respond extremely well to treatment (see discussion). With this patient with tardive dystonia excluded from the analysis, secondary aetiology was predictive of a poorer response to DBS, with the improvement in BFM-S reduced by 16.1 percentage points $(p=0.013)$. The improvement in BFM-D was lowered by 7.3 percentage points, however this was not significant $(p=0.48)$.

\section{Effect of phenotype}

Dystonia of torsional and myoclonic phenotypes responded well to DBS. Having a myoclonic rather than torsional phenotype resulted in a trend towards better results but this was non-significant.
Effect of adult versus paediatric onset disease

A disease onset in adulthood rather than childhood made only a minor and non-significant difference to the size of the effect of treatment with DBS on BFM-S and BFM-D.

\section{Effect of disease duration prior to treatment}

Patients were grouped into those who had dystonia for up to 10 years, $10-20$ years or over 20 years prior to treatment with DBS. A longer duration of disease prior to treatment had no significant impact on the efficacy of DBS. Those in the group with the shortest duration (up to 10 years) had higher baseline scores than those in the longer duration groups but the percentage improvement in BFM scores was similar.

\section{DISCUSSION}

This is the largest published series of outcomes in GPi DBS for generalised dystonia. In line with several other studies we confirmed that bilateral GPi DBS has a sustained beneficial effect on generalised dystonia. In this group of 60 patients there was a highly significant improvement in the severity and the disability scores of the BFMDRS by 6 months postoperatively $(p<0.001$ for BFM-S and BFM-D), and this effect did not wane at all over subsequent follow-up to 5 years.

Patients with DYT mutations obtained a significantly greater benefit from DBS than those without. The three with DYT6 or DYT11 mutations showed a similar response to those with DYT1 mutations (mean improvements in BFM-S at 1 year of $52 \%$ for DYT6/ DYT11 and 49\% for DYT1). Indeed one of the three (with DYT11) showed an 81\% improvement in BFM-S; the patients with DYT6 showed 23\% and 52\% improvements. The patient with DYT11 (myoclonus dystonia) also saw an
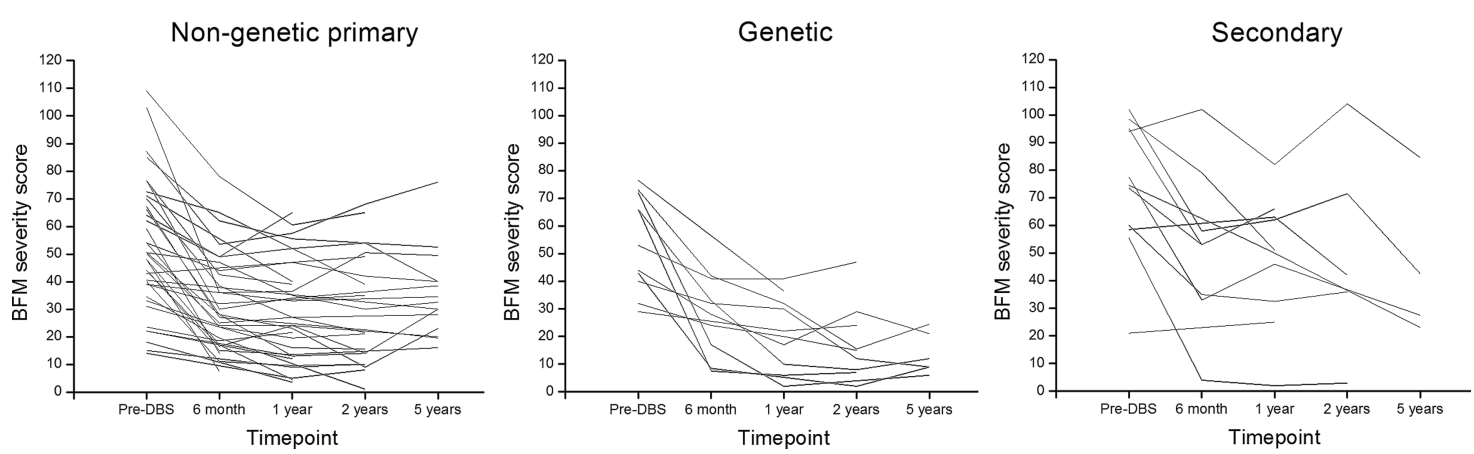

Figure 3 Individual outcomes: changes in Burke-Fahn-Marsden (BFM) severity score for non-genetic primary generalised dystonia (left), DYT gene mutation associated dystonia (centre) and secondary generalised dystonia (right). DBS, deep brain stimulation. 


\begin{tabular}{|c|c|c|c|c|}
\hline & \multicolumn{4}{|c|}{$\begin{array}{l}\text { Percentage change in BFM scores } \\
\text { (negative indicates improvement) }\end{array}$} \\
\hline & \multicolumn{2}{|c|}{ Severity score } & \multicolumn{2}{|c|}{ Disability score } \\
\hline & Estimate & p value & Estimate & p value \\
\hline \multicolumn{5}{|l|}{ Reference group: } \\
\hline Childhood-onset primary generalised dystonia of torsional phenotype and less than 10 years duration, at 6 months follow-up & -40.3 & $<0.001$ & -28.0 & 0.003 \\
\hline \multicolumn{5}{|l|}{ Additional effect of changes in fixed variables, relative to reference group above (negative indicates improvement): } \\
\hline \multicolumn{5}{|l|}{ Later time points } \\
\hline 1 year & -1.0 & 0.81 & -6.1 & 0.39 \\
\hline 2 years & -5.5 & 0.31 & +5.3 & 0.65 \\
\hline 5 years & -8.3 & 0.17 & -3.6 & 0.75 \\
\hline \multicolumn{5}{|l|}{ Different aetiology } \\
\hline DYT mutation & -14.8 & 0.025 & -20.7 & 0.031 \\
\hline Secondary & +6.3 & 0.40 & +2.4 & 0.82 \\
\hline Secondary (tardive dystonia excluded) & +16.1 & 0.013 & +7.3 & 0.48 \\
\hline Myoclonic phenotype & -11.1 & 0.11 & -18.6 & 0.11 \\
\hline Adult onset & +4.4 & 0.46 & -8.3 & 0.38 \\
\hline \multicolumn{5}{|l|}{ Increased duration } \\
\hline $10-20$ years & -6.1 & 0.36 & +8.2 & 0.36 \\
\hline $20+$ years & +4.4 & 0.48 & +15.9 & 0.091 \\
\hline
\end{tabular}

improvement in myoclonic symptoms, although this was not quantified, The literature concerning the response to DBS in patients with mutations at loci other than DYT1 is sparse, however in the small numbers with DYT6 ${ }^{27} 28$ and DYT11 ${ }^{29}$ that have been reported, the results have been encouraging. It is clear at least that individual cases with DYT6 or DYT11 mutations have the potential to respond as well to treatment as the typical patient with DYT1 does. Interestingly, possession of a DYT mutation made a much greater difference to the response seen in BFM-D (where the response was $175 \%$ of the non-DYT response) than BFM-S (where the response was $136 \%$ of the non-DYT response).

Overall, the 12 secondary dystonia cases tended to have a poorer outcome than the non-genetic primary cases. On initial analysis this was not statistically significant, but this analysis was distorted by the single tardive dystonia case whose BFM-S improved by over $90 \%$, while no other case responded better than $50 \%$. This is in line with the exceptionally good results seen in published series of GPi DBS in patients with tardive dystonia. ${ }^{30} 31$ With this patient excluded, the secondary cases showed a significantly poorer response in BFM-S than the non-genetic primary cases $(p=0.013)$. However, the reduction amounted to less than half of the overall treatment effect and there were still highly significant improvements in BFM-S $(p=0.0033$ at 1 year) and BFM-D ( $p=0.0029$ at 1 year) in this group. Of the metabolic abnormalities, the patient with glutaric aciduria did not respond to DBS, while the patient with iron deposition responded well (BFM-S improved $44 \%$ at 6 months and $50 \%$ at 5 years). The patient with pantothenate kinase-associated neurodegeneration had a $42 \%$ improvement in BFM-S at 6 months (no later follow-up data are available).

The efficacy of treatment was unaffected by the age group of disease onset (adult vs paediatric) or the disease phenotype (torsional vs myoclonic).

Previous series have differed as to whether the duration of disease prior to treatment is ${ }^{1922} 23$ or is not ${ }^{412}$ predictive of response. One might intuitively expect that more established disease would be harder to treat, but we did not find this. Our patients with disease duration of less than 10 years had higher baseline BFM scores than those treated later in their disease course (suggesting that more severe cases tended to reach neurosurgical treatment earlier) but the proportionate improvement in BFM with treatment was similar. It must be borne in mind however that there are only five patients in this study whose disease duration was shorter than 5 years and no patient had dystonia for less than 2 years. It may well be that duration is an important factor at shorter timescales, for example, dystonia of 1 year duration may respond better than 5 years, and the results presented here should not be taken as an indication that it does not matter how long dystonia is left before it is treated. Indeed a study of outcomes after DBS in a large number of children with dystonia, whose disease durations were much shorter than those presented here, ${ }^{23}$ found that longer duration of disease (expressed as a proportion of the child's life for which they had had dystonia) was a significant negative predictor of response.

Eight patients $(13 \%)$ in this study had an infection of their system at some point during follow-up. In every case, infection followed IPG replacement rather than occurring at primary surgery. A substantially higher infection rate at IPG replacement compared with primary surgery has also been reported by others. ${ }^{32}$ This is a powerful argument for the use of rechargeable IPGs, and that is now our routine practice.

The main limitations of this study are that it is retrospective (although data were collected prospectively) and follow-up is limited to 5 years.

While the question of which patient groups respond best to DBS is important, the main message of this study is that all subgroups analysed, whether primary idiopathic, genetic or secondary, paediatric or adult onset, torsional or myoclonic phenotype, and whatever the duration of disease, had a substantial reduction in severity scores compared with preoperative clinical 
assessments, and importantly also had a reduction in the selfreported disability score. There was no group who consistently failed to respond to DBS. We can see no basis for excluding any of the categories of patients with generalised dystonia we examined from consideration for DBS.

Contributors JJF, TZA, ALG, FR, NdP conceived the study and performed surgery. CJ, BF, CF performed and analysed Burke-Fahn-Marsden assessments. JJF, FR, NdP drafted the manuscript which was reviewed critically by all the other authors. All authors approved the final manuscript prior to submission.

\section{Competing interests None.}

Ethics approval This study used only data collected as part of routine clinical practice and no patient identifiable information is presented. No specific ethical approval was required.

Provenance and peer review Not commissioned; externally peer reviewed.

\section{REFERENCES}

1 Charlesworth G, Bhatia KP, Wood NW. The genetics of dystonia: new twists in an old tale. Brain 2013;136:2017-37.

2 Mundinger F. [New stereotactic treatment of spasmodic torticollis with a brain stimulation system (author's trans))]. Med Klin 1977;72:1982-6.

3 Vidailhet $\mathrm{M}$, Vercueil $\mathrm{L}$, Houeto $\mathrm{JL}$, et al. Bilateral deep-brain stimulation of the globus pallidus in primary generalized dystonia. N Engl J Med 2005;352:459-67.

4 Kupsch A, Benecke R, Muller J, et al. Pallidal deep-brain stimulation in primary generalized or segmental dystonia. N Eng/ J Med 2006;355:1978-90.

5 Vidailhet M, Vercueil L, Houeto JL, et al. Bilateral, pallidal, deep-brain stimulation in primary generalised dystonia: a prospective 3 year follow-up study. Lancet Neurol 2007;6:223-9.

6 Volkmann J, Wolters A, Kupsch A, et al. Pallidal deep brain stimulation in patients with primary generalised or segmental dystonia: 5 -year follow-up of a randomised trial. Lancet Neurol 2012;11:1029-38.

7 Coubes $\mathrm{P}, \mathrm{Cif} \mathrm{L}$, El Fertit $\mathrm{H}$, et al. Electrical stimulation of the globus pallidus internus in patients with primary generalized dystonia: long-term results, J Neurosurg 2004;101:189-94.

8 Isaias IU, Alterman RL, Tagliati M. Deep brain stimulation for primary generalized dystonia: long-term outcomes. Arch Neurol 2009;66:465-70.

9 Cif $\mathrm{L}$, El Fertit $\mathrm{H}$, et al. Treatment of dystonic syndromes by chronic electrical stimulation of the internal globus pallidus. J Neurosurg Sci 2003;47:52-5.

10 Bittar RG, Yianni J, Wang S, et al. Deep brain stimulation for generalised dystonia and spasmodic torticollis. J Clin Neurosci 2005:12:12-16.

11 Coubes P, Roubertie A, Vayssiere N, et al. Treatment of DYT1-generalised dystonia by stimulation of the internal globus pallidus. Lancet 2000;355:2220-1.

12 Valldeoriola F, Regidor I, Minguez-Castellanos A, et al. Efficacy and safety of pallidal stimulation in primary dystonia: results of the Spanish multicentric study. J Neurol Neurosurg Psychiatry 2010;81:65-9.
13 Panov F, Gologorsky Y, Connors G, et al. Deep brain stimulation in DYT1 dystonia: a 10-year experience. Neurosurgery 2013;73:86-93.

14 Vercueil L, Krack P, Pollak P. Results of deep brain stimulation for dystonia: a critica reappraisal. Mov Disord 2002;17(Suppl 3):S89-93.

15 Krause $\mathrm{M}$, Fogel $\mathrm{W}$, Kloss $\mathrm{M}$, et al. Pallidal stimulation for dystonia. Neurosurgery 2004;55:1361-8; discussion 8-70.

16 Eltahawy HA, Saint-Cyr J, Giladi N, et al. Primary dystonia is more responsive than secondary dystonia to pallidal interventions: outcome after pallidotomy or pallidal deep brain stimulation. Neurosurgery 2004;54:613-19; discussion 9-21.

17 Yianni J, Bain P, Giladi N, et al. Globus pallidus internus deep brain stimulation for dystonic conditions: a prospective audit. Mov Disord 2003;18:436-42.

18 Tisch S, Zrinzo L, Limousin P, et al. Effect of electrode contact location on clinical efficacy of pallidal deep brain stimulation in primary generalised dystonia. J Neurol Neurosurg Psychiatry 2007:78:1314-19.

19 Isaias IU, Alterman RL, Tagliati M. Outcome predictors of pallidal stimulation in patients with primary dystonia: the role of disease duration. Brain 2008;131:1895-902.

20 Vidailhet $\mathrm{M}$, Jutras MF, Grabli $\mathrm{D}$, et al. Deep brain stimulation for dystonia. J Neurol Neurosurg Psychiatry 2013;84:1029-42.

21 Andrews C, Aviles-Olmos I, Hariz M, et al. Which patients with dystonia benefit from deep brain stimulation? A metaregression of individual patient outcomes. I Neurol Neurosurg Psychiatry 2010;81:1383-9.

22 Mehrkens JH, Botzel K, Steude U, et al. Long-term efficacy and safety of chronic globus pallidus internus stimulation in different types of primary dystonia. Stereotact Funct Neurosurg 2009:87:8-17.

23 Lumsden DE, Kaminska M, Gimeno $H$, et al. Proportion of life lived with dystonia inversely correlates with response to pallidal deep brain stimulation in both primary and secondary childhood dystonia. Dev Med Child Neurol 2013;55:567-74.

24 Fahn S. Concept and classification of dystonia. Adv Neurol 1988;50:1-8.

25 Burke RE, Fahn $S$, Marsden CD, et al. Validity and reliability of a rating scale for the primary torsion dystonias. Neurology 1985;35:73-7.

26 Hyam JA, de Pennington N, Joint $\mathrm{C}$, et al. Maintained deep brain stimulation for severe dystonia despite infection by using externalized electrodes and an extracorporeal pulse generator. J Neurosurg 2010;113:630-3

27 Panov F, Tagliati M, Ozelius LJ, et al. Pallidal deep brain stimulation for DYT6 dystonia. J Neurol Neurosurg Psychiatry 2012;83:182-7.

28 Groen JL, Ritz K, Contarino MF, et al. DYT6 dystonia: mutation screening, phenotype, and response to deep brain stimulation. Mov Disord 2010;25: 2420-7.

29 Gruber D, Kuhn AA, Schoenecker T, et al. Pallidal and thalamic deep brain stimulation in myoclonus-dystonia. Mov Disord 2010;25:1733-43.

30 Gruber D, Trottenberg T, Kivi A, et al. Long-term effects of pallidal deep brain stimulation in tardive dystonia. Neurology 2009;73:53-8.

31 Capelle HH, Blahak C, Schrader C, et al. Chronic deep brain stimulation in patients with tardive dystonia without a history of major psychosis. Mov Disord 2010;25:1477-81.

32 Pepper J, Zrinzo L, Mirza B, et al. The risk of hardware infection in deep brain stimulation surgery is greater at impulse generator replacement than at the primary procedure. Stereotact Funct Neurosurg 2013;91:56-65. 